\title{
New Regularized Algorithms for Transductive Learning
}

\author{
Partha Pratim Talukdar and Koby Crammer \\ Computer \& Information Science Department \\ University of Pennsylvania \\ Philadelphia, PA 19104 \\ \{partha, crammer\}@is. upenn.edu
}

\begin{abstract}
We propose a new graph-based label propagation algorithm for transductive learning. Each example is associated with a vertex in an undirected graph and a weighted edge between two vertices represents similarity between the two corresponding example. We build on Adsorption, a recently proposed algorithm and analyze its properties. We then state our learning algorithm as a convex optimization problem over multi-label assignments and derive an efficient algorithm to solve this problem. We state the conditions under which our algorithm is guaranteed to converge. We provide experimental evidence on various real-world datasets demonstrating the effectiveness of our algorithm over other algorithms for such problems. We also show that our algorithm can be extended to incorporate additional prior information, and demonstrate it with classifying data where the labels are not mutually exclusive.
\end{abstract}

Keywords: label propagation, transductive learning, graph based semi-supervised learning.

\section{Introduction}

Supervised machine learning methods have achieved considerable success in a wide variety of domains ranging from Natural Language Processing, Speech Recognition to Bioinformatics. Unfortunately, preparing labeled data for such methods is often expensive and time consuming, while unlabeled data are widely available in many cases. This was the major motivation that led to the development of semi-supervised algorithms which learn from limited amounts of labeled data and vast amounts of freely available unannotated data.

Recently, graph based semi-supervised algorithms have achieved considerable attention [2 7]11/14]17]. Such methods represent instances as vertices in a graph with edges between vertices encoding similarities between them. Graph-based semi-supervised algorithms often propagate the label information from the few labeled vertices to the entire graph. Most of the algorithms tradeoff between accuracy (initially labeled nodes should retain those labels, relaxations allowed by some methods) with smoothness (adjacent vertices in the graph should be assigned similar labels). Most algorithms only output label information to the unlabeled data in a transductive setting, while some algorithms are designed for the semi-supervised framework and build a classification model which can be applied to out-of-sample examples. 
Adsorption [1] is one such recently proposed graph based semi-supervised algorithm which has been successfully used for different tasks, such as recommending YouTube videos to users [1] and large scale assignment of semantic classes to entities within Information Extraction [13]. Adsorption has many desirable properties: it can perform multiclass classification, it can be parallelized and hence can be scaled to handle large data sets which is of particular importance for semi-supervised algorithms. Even though Adsorption works well in practice, to the best of our knowledge it has never been analyzed before and hence our understanding of it is limited. Hoping to fill this gap, we make the following contributions in this paper:

- We analyze the Adsorption algorithm [1] and show that there does not exist an objective function whose local optimization would be the output of the Adsorption algorithm.

- Motivated by this negative result, we propose a new graph based semi-supervised algorithm (Modified Adsorption, MAD), which shares Adsorption's desirable properties, yet with some important differences.

- We state the learning problem as an optimization problem and develop efficient (iterative) methods to solve it. We also list the conditions under which the optimization algorithm - MAD - is guaranteed to converge.

- The transition to an optimization based learning algorithm provides a flexible and general framework that enables us to specify a variety requirements. We demonstrate this framework using data with non-mutually exclusive labels, resulting in the Modified Adsorption for Dependent Labels (MADDL, pronounced medal) algorithm.

- We provide experimental evidence demonstrating the effectiveness of our proposed algorithm on various real world datasets.

\section{Adsorption Algorithm}

Adsorption [1] is a general algorithmic framework for transductive learning where the learner is often given a small set of labeled examples and a very large set of unlabeled examples. The goal is to label all the unlabeled examples, and possibly under the assumption of label-noise, also to relabel the labeled examples.

As many other related algorithms [17|12]5], Adsorption assumes that the learning problem is given in a graph form, where examples or instances are represented as nodes or vertices and edges code similarity between examples. Some of the nodes are associated with a pre-specified label, which is correct in the noise-free case, or can be subject to label-noise. Additional information can be given in the form of weights over the labels. Adsorption propagates label-information from the labeled examples to the entire set of vertices via the edges. The labeling is represented using a non-negative score for each label, with high score for some label indicating high-association of a vertex (or its corresponding instance) with that label. If the scores are additively normalized they can be thought of as a conditional distribution over the labels given the node (or example) identity.

More formally, Adsorption is given an undirected graph $G=(V, E, W)$, where a node $v \in V$ corresponds to an example, an edge $e=(a, b) \in V \times V$ indicates that the 
label of the two vertices $a, b \in V$ should be similar and the weight $W_{a b} \in \mathbb{R}_{+}$reflects the strength of this similarity.

We denote the total number of examples or vertices by $n=|V|$, by $n_{l}$ the number of examples for which we have prior knowledge of their label and by $n_{u}$ the number of unlabeled examples to be labeled. Clearly $n_{l}+n_{u}=n$. Let $\mathcal{L}$ be the set of possible labels, their total number is denoted by $m=|\mathcal{L}|$ and without loss of generality we assume that the possible labels are $\mathcal{L}=\{1 \ldots m\}$. Each instance $v \in V$ is associated with two row-vectors $\mathbf{Y}_{v}, \hat{\mathbf{Y}}_{v} \in \mathbb{R}_{+}^{m}$. The $1 t h$ element of the vector $\mathbf{Y}_{v}$ encodes the prior knowledge for vertex $v$. The higher the value of $\mathbf{Y}_{v l}$ the stronger we a-priori believe that the label of $v$ should be $l \in \mathcal{L}$ and a value of zero $\mathbf{Y}_{v l}=0$ indicates no prior about the label $l$ for vertex $v$. Unlabeled examples have all their elements set to zero, that is $\mathbf{Y}_{v l}=0$ for $l=1 \ldots m$. The second vector $\hat{\mathbf{Y}}_{v} \in \mathbb{R}_{+}^{m}$ is the output of the algorithm, using similar semantics as $\mathbf{Y}_{v}$. For example, a high value of $\hat{\mathbf{Y}}_{v l}$ indicates that the algorithm believes that the vertex $v$ should have the label $l$. We denote by $\mathbf{Y}, \hat{\mathbf{Y}} \in \mathbb{R}_{+}^{n \times m}$ the matrices whose rows are $\mathbf{Y}_{v}$ and $\hat{\mathbf{Y}}_{v}$ respectively. Finally, we denote by $\mathbf{0}_{d}$ the all-zeros row vector of dimension $d$.

\subsection{Random-Walk View}

The Adsorption algorithm can be viewed as a controlled random walk over the graph $G$. The control is formalized via three possible actions: inject, continue and abandon (denoted by inj, cont, abnd) with pre-defined probabilities $p_{v}^{\mathrm{i} n j}, p_{v}^{\mathrm{cont}}, p_{v}^{\mathrm{abnd}} \geq$ 0 per vertex $v \in V$. Clearly their sum is unit: $p_{v}^{\mathrm{i} n j}+p_{v}^{\text {cont }}+p_{v}^{\text {abnd }}=1$. To label any vertex $v \in V$ (either labeled or unlabeled) we initiate a random-walk starting at $v$ facing three options: with probability $p_{v}^{\mathrm{i} n j}$ the random-walk stops and return (i.e. inject) the pre-defined vector information $\mathbf{Y}_{v}$. We constrain $p_{v}^{\mathrm{i} n j}=0$ for unlabeled vertices $v$. Second, with probability $p_{v}^{\text {abnd }}$ the random-walk abandons the labeling process and return the all-zeros vector $\mathbf{0}_{m}$. Third, with probability $p_{v}^{\text {cont }}$ the random-walk continues to one of v's neighbors $v^{\prime}$ with probability proportional to $W_{v^{\prime} v} \geq 0$. Note that by definition $W_{v^{\prime} v}=0$ if $\left(v, v^{\prime}\right) \notin E$. We summarize the above process with the following set of equations. The transition probabilities are,

$$
\operatorname{Pr}\left[v^{\prime} \mid v\right]=\left\{\begin{array}{ll}
\frac{W_{v^{\prime} v}}{\sum_{u:(u, v) \in E} W_{u v}}\left(v^{\prime}, v\right) \in E \\
0 \quad \text { otherwise }
\end{array} .\right.
$$

The (expected) score $\hat{\mathbf{Y}}_{v}$ for node $v \in V$ is given by,

$$
\hat{\mathbf{Y}}_{v}=p_{v}^{\mathrm{i} n j} \times \mathbf{Y}_{v}+p_{v}^{\mathrm{cont}} \times \sum_{v^{\prime}:\left(v^{\prime}, v\right) \in E} \operatorname{Pr}\left[v^{\prime} \mid v\right] \hat{\mathbf{Y}}_{v^{\prime}}+p_{v}^{\mathrm{a} b n d} \times \mathbf{0}_{m}
$$

\subsection{Averaging View}

For this view we add a designated symbol called the dummy label denoted by $\nu \notin \mathcal{L}$. This additional label explicitly encodes ignorance about the correct label and it means 


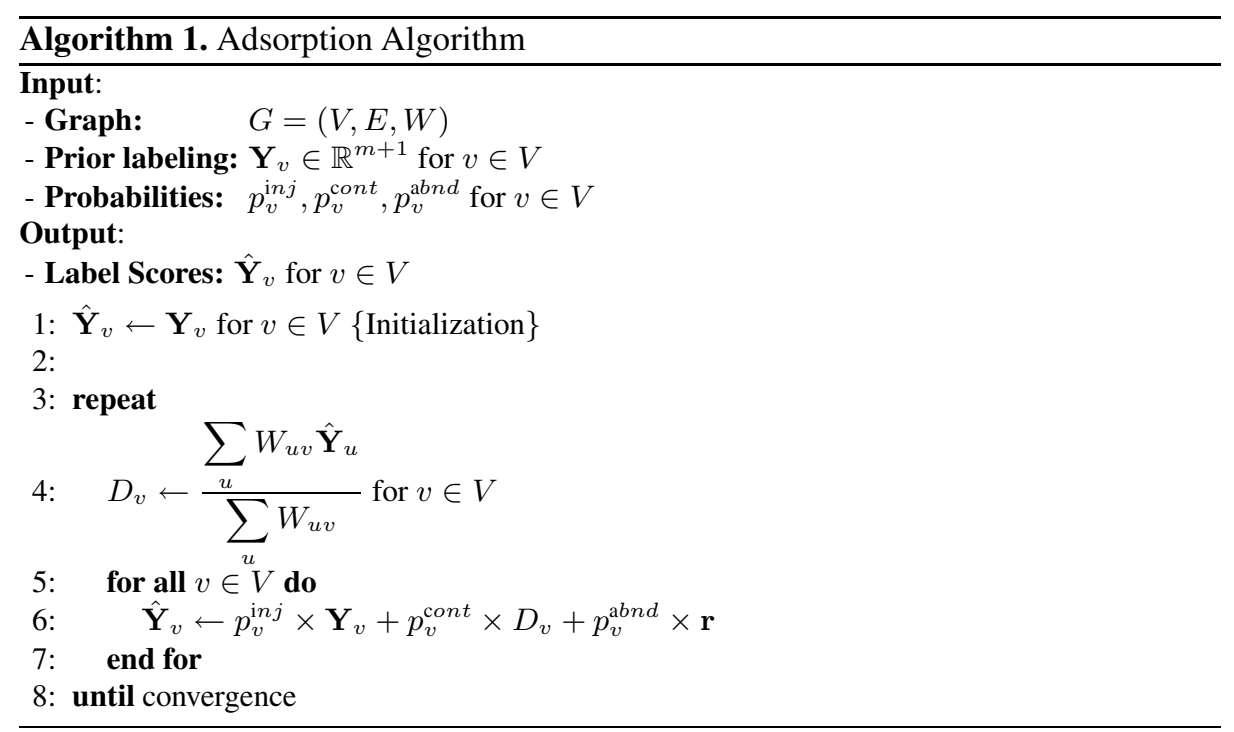

that a dummy label can be used instead. Explicitly, we add an additional column to all the vectors defined above, and have that $\mathbf{Y}_{v}, \hat{\mathbf{Y}}_{v} \in \mathbb{R}_{+}^{m+1}$ and $\mathbf{Y}, \hat{\mathbf{Y}} \in \mathbb{R}_{+}^{n \times(m+1)}$. We set $\mathbf{Y}_{v \nu}=0$, that is, a-priori no vertex is associated with the dummy label, and replace the zero vector $\mathbf{0}_{m}$ with the vector $\mathbf{r} \in \mathbb{R}_{+}^{m+1}$ where $\mathbf{r}_{l}=0$ for $l \neq \nu$ and $\mathbf{r}_{\nu}=1$. In words, if the random-walk is abandoned, then the corresponding labeling vector is zero for all true labels in $\mathcal{L}$, and an arbitrary value of unit for the dummy label $\nu$. This way, there is always positive score for at least one label, the ones in $\mathcal{L}$ or the dummy label.

The averaging view then defines a set of fixed-point equations to update the predicted labels. A summary of the equations appears in Algorithm 1 The algorithm is run until convergence which is achieved when the label distribution on each node ceases to change within some tolerance value. Since Adsorption is memoryless, it scales to tens of millions of nodes with dense edges and can be easily parallelized [1].

Baluja et. al. [1] show that up to the additional dummy label, these two views are equivalent. It remains to specify the values of $p_{v}^{\mathrm{i} n j}, p_{v}^{\mathrm{cont}}$ and $p_{v}^{\mathrm{a} b n d}$. For the experiments reported in Section 6, we set their value using the following heuristics (adapted from Baluja et. al. [1]) which depends on a parameter $\beta$ which we set to $\beta=2$. For each node $v$ we define two quantities: $c_{v}$ and $d_{v}$ and define

$$
p_{v}^{\text {cont }} \propto c_{v} \quad ; \quad p_{v}^{\text {inj }} \propto d_{v}
$$

The first quantity $c_{v} \in[0,1]$ is monotonically decreasing with the number of neighbors for node $v$ in the graph $G$. Intuitively, the higher the value of $c_{v}$, the lower the number of neighbors of vertex $v$ and higher the information they contain about the labeling of $v$. The other quantity $d_{v} \geq 0$ is monotonically increasing with the entropy (for labeled vertices), and in this case we prefer to use the prior-information rather than the computed quantities from the neighbors. 
Specifically we first compute the entropy of the transition probabilities for each node,

$$
H[v]=-\sum_{u} \operatorname{Pr}[u \mid v] \log \operatorname{Pr}[u \mid v],
$$

and then pass it through the following monotonically decreasing function,

$$
f(x)=\frac{\log \beta}{\left.\log \left(\beta+e^{x}\right)\right)} .
$$

Note that $f(0)=\log (\beta) / \log (\beta+1)$ and that $f(x)$ goes to zero, as $x$ goes to infinity. We define,

$$
c_{v}=f(H[v])
$$

Next we define,

$$
d_{v}=\left\{\begin{aligned}
\left(1-c_{v}\right) \times \sqrt{H[v]} & \text { the vertex } \mathrm{v} \text { is labeled } \\
0 & \text { the vertex } \mathrm{v} \text { is unlabled }
\end{aligned}\right.
$$

Finally, to ensure proper normalization of $p_{v}^{\mathrm{cont}}, p_{v}^{\mathrm{i} n j}$ and $p_{v}^{\mathrm{a} b n d}$, we define,

$$
z_{v}=\max \left(c_{v}+d_{v}, 1\right)
$$

and

$$
p_{v}^{\mathrm{cont}}=\frac{c_{v}}{z_{v}} \quad ; \quad p_{v}^{\mathrm{i} n j}=\frac{d_{v}}{z_{v}} \quad ; \quad p_{v}^{\mathrm{a} b n d}=1-p_{v}^{\mathrm{cont}}-p_{v}^{\mathrm{i} n j} .
$$

Thus, abandonment occurs only when the continuation and injection probabilities are low enough. This is most likely to happen at unlabeled nodes with high degree. Once the random walk reaches such a node $(v)$, the walk is terminated with probability $p_{v}^{\mathrm{a} b n d}$. This, in effect, prevents the Adsorption algorithm from propagating information through high degree nodes. We note that the probabilities $p_{v}^{\mathrm{i} n j}, p_{v}^{\mathrm{cont}}$ and $p_{v}^{\mathrm{a} b n d}$ for node $v$ may be set with heuristics other than the fan-out entropy heuristics shown above to suit specific application contexts.

\section{Analysis of the Adsorption Algorithm}

Our next goal is to find an objective function that the Adsorption algorithm minimizes. Our starting point is line 6 of Algorithm 1 We note that when the algorithm converges, both sides of the assignment operator equal each other before the assignment takes place. Thus when the algorithm terminates, we have for all $v \in V$ :

$$
\hat{\mathbf{Y}}_{v}=p_{v}^{\mathrm{i} n j} \times \mathbf{Y}_{v}+p_{v}^{\mathrm{cont}} \times \frac{1}{N_{v}} \sum_{u} W_{u v} \hat{\mathbf{Y}}_{u}+p_{i}^{\mathrm{a} b n d} \times \mathbf{r}
$$

where

$$
N_{v}=\sum_{v^{\prime}} W_{v^{\prime} v}
$$


The last set of equalities is equivalent to,

$$
G_{v}\left(\left\{\hat{\mathbf{Y}}_{u}\right\}_{u \in V}\right)=0 \text { for } v \in V
$$

where we define,

$$
G_{v}\left(\left\{\hat{\mathbf{Y}}_{u}\right\}_{u \in V}\right)=p_{v}^{\mathrm{i} n j} \times \mathbf{Y}_{v}+p_{v}^{\mathrm{cont}} \times \frac{1}{N_{v}} \sum_{u} W_{u v} \hat{\mathbf{Y}}_{u}+p_{i}^{\mathrm{a} b n d} \times \mathbf{r}-\hat{\mathbf{Y}}_{v} .
$$

Now, if the Adsorption algorithm was minimizing some objective function (denoted by $\left.\mathcal{Q}\left(\left\{\hat{\mathbf{Y}}_{u}\right\}_{u \in V}\right)\right)$, the termination condition of Eq. (3) was in fact a condition on the vector of its partial derivatives where we would identify

$$
G_{v}=\frac{\partial}{\partial \hat{\mathbf{Y}}_{v}} \mathcal{Q}
$$

Since the functions $G_{v}$ are linear (and thus has continuous derivatives), necessary conditions for the existence of a function $\mathcal{Q}$ such that (4) holds is that the derivatives of $G_{v}$ are symmetric [8], that is,

$$
\frac{\partial}{\partial \hat{\mathbf{Y}}_{v}} G_{u}=\frac{\partial}{\partial \hat{\mathbf{Y}}_{u}} G_{v}
$$

Computing and comparing the derivatives we get,

$$
\frac{\partial}{\partial \hat{\mathbf{Y}}_{u}} G_{v}=p_{v}^{\mathrm{cont}}\left(\frac{W_{u v}}{N_{v}}-\delta_{u, v}\right) \neq p_{u}^{\mathrm{cont}}\left(\frac{W_{v u}}{N_{u}}-\delta_{u, v}\right)=\frac{\partial}{\partial \hat{\mathbf{Y}}_{v}} G_{u},
$$

which is true since in general $N_{u} \neq N_{v}$ and $p_{v}^{\text {cont }} \neq p_{u}^{\text {cont }}$. We conclude:

Theorem 1. There does not exists a function $\mathcal{Q}$ with continuous second partial derivatives such that the Adsorption algorithm convergences when gradient of $\mathcal{Q}$ are equal to zero.

In other words, we searched for a (well-behaved) function $\mathcal{Q}$ such that its local optimal would be the output of the Adsorption algorithm, and showed that this search will always fail. We use this negative results to define a new algorithm, which builds on the Adsorption algorithm and is optimizing a function of the unknowns $\hat{\mathbf{Y}}_{v}$ for $v \in V$.

\section{New Algorithm: Modified Adsorption (MAD)}

Our starting point is Sec. 2.2 where we assume to have been given a weighted-graph $G=(V, E, W)$ and a matrix $\mathbf{Y} \in \mathbb{R}_{+}^{n \times(m+1)}$ and are seeking for a labeling-matrix $\hat{\mathbf{Y}} \in \mathbb{R}_{+}^{n \times(m+1)}$. In this section it is more convenient to decompose the matrices $\mathbf{Y}$ and $\hat{\mathbf{Y}}$ into their columns, rather than rows. Specifically, we denote by $\mathbf{Y}_{l} \in \mathbb{R}_{+}^{n}$ the $1 t h$ column of $\mathbf{Y}$ and similarly by $\hat{\mathbf{Y}}_{l} \in \mathbb{R}_{+}^{n}$ the $1 t h$ column of $\hat{\mathbf{Y}}$. We distinguish the rows and columns of the matrices $\mathbf{Y}$ and $\hat{\mathbf{Y}}$ using their indices, the columns are indexed with the label index $l$, while the rows are indexed are with a vertex index $v$ (or $u$ ). 
We build on previous research [3|17|11] and construct an objective that reflects three requirements as follows. First, for the labeled vertices we like the output of the algorithm to be close to the a-priori given labels, that is $\mathbf{Y}_{v} \approx \hat{\mathbf{Y}}_{v}$. Second, for pair of vertices that are close according to the input graph, we would like their labeling to be close, that is $\hat{\mathbf{Y}}_{u} \approx \hat{\mathbf{Y}}_{v}$ if $W_{u v}$ is large. Third, we want the output to be as uninformative as possible, this serves as additional regularization, that is $\hat{\mathbf{Y}}_{v} \approx \mathbf{r}$. We now further develop the objective in light of the three requirements.

We use the Euclidian distance to measure discrepancy between two quantities, and start with the first requirement above,

$$
\begin{aligned}
\sum_{v} p_{v}^{\mathrm{i} n j} \sum_{l}\left(\mathbf{Y}_{v l}-\hat{\mathbf{Y}}_{v l}\right)^{2} & =\sum_{l} \sum_{v} p_{v}^{\mathrm{i} n j}\left(\mathbf{Y}_{v l}-\hat{\mathbf{Y}}_{v l}\right)^{2} \\
& =\sum_{l}\left(\mathbf{Y}_{l}-\hat{\mathbf{Y}}_{l}\right)^{\top} \mathbf{S}\left(\mathbf{Y}_{l}-\hat{\mathbf{Y}}_{l}\right)
\end{aligned}
$$

where we define the diagonal matrix $\mathbf{S} \in \mathbb{R}^{n \times n}$ and $\mathbf{S}_{v v}=p_{v}^{\mathrm{i} n j}$ if vertex $v$ is labeled and $\mathbf{S}_{v v}=0$ otherwise. The matrix $\mathbf{S}$ captures the intuition that for different vertices we enforce the labeling of the algorithm to match the a-priori labeling with different extent.

Next, we modify the similarity weight between vertices to take into account the difference in degree of various vertices. In particular we define $\mathbf{W}_{v u}^{\prime}=p_{v}^{\text {cont }} \times \mathbf{W}_{v u}$. Thus, a vertex $u$ will not be similar to a vertex $v$ if either the input weights $\mathbf{W}_{v u}$ are low or the vertex $v$ has a large-degree ( $p_{v}^{\text {cont }}$ is low). We write the second requirement as,

$$
\begin{aligned}
& \sum_{v, u} \mathbf{W}_{v u}^{\prime}\left\|\hat{\mathbf{Y}}_{v}-\hat{\mathbf{Y}}_{u}\right\|^{2}=\sum_{v, u} \mathbf{W}_{v u}^{\prime} \sum_{l}\left(\hat{\mathbf{Y}}_{v l}-\hat{\mathbf{Y}}_{u l}\right)^{2}=\sum_{l} \sum_{v, u} \mathbf{W}_{v u}^{\prime}\left(\hat{\mathbf{Y}}_{v l}-\hat{\mathbf{Y}}_{u l}\right)^{2} \\
= & \sum_{l} \sum_{v}\left(\sum_{u} \mathbf{W}_{v u}^{\prime}\right)\left\|\hat{\mathbf{Y}}_{v l}\right\|^{2}+\sum_{l} \sum_{u}\left(\sum_{v} \mathbf{W}_{v u}^{\prime}\right)\left\|\hat{\mathbf{Y}}_{u l}\right\|^{2}-2 \sum_{l} \sum_{u, v} \mathbf{W}_{v u}^{\prime} \hat{\mathbf{Y}}_{u l} \hat{\mathbf{Y}}_{v l} \\
= & \sum_{l} \hat{\mathbf{Y}}_{l}^{\top} \mathbf{L} \hat{\mathbf{Y}}_{l},
\end{aligned}
$$

where,

$$
\mathbf{L}=\mathbf{D}+\overline{\mathbf{D}}-\mathbf{W}^{\prime}-\mathbf{W}^{\prime \top}
$$

and $\mathbf{D}, \overline{\mathbf{D}}$ are $n \times n$ diagonal matrices with

$$
\mathbf{D}_{v v}=\sum_{u} \mathbf{W}_{u v}^{\prime} \quad, \quad \overline{\mathbf{D}}_{v v}=\sum_{u} \mathbf{W}_{v u}^{\prime} .
$$

Finally we define the matrix $\mathbf{R} \in \mathbb{R}_{+}^{n \times(m+1)}$ where the vth row of $\mathbf{R}$ equals $p_{v}^{\mathrm{a} b n d} \times \mathbf{r}$ (we define $\mathbf{r}$ in $\mathrm{Sec} 2.2$ ). In other words the first $m$ columns of $\mathbf{R}$ equal zero, and the last $\left(\mathrm{m}+1\right.$ th column) equal the elements of $p_{v}^{\mathrm{a} b n d}$. The third requirement above is thus written as,

$$
\sum_{v l}\left(\hat{\mathbf{Y}}_{v l}-\mathbf{R}_{v l}\right)^{2}=\sum_{l}\left\|\hat{\mathbf{Y}}_{l}-\mathbf{R}_{l}\right\|^{2}
$$


We combine the three terms above into a single objective (which we would like to minimize), giving to each term a different importance using the weights $\mu_{1}, \mu_{2}, \mu_{3}$.

$$
C(\hat{\mathbf{Y}})=\sum_{l}\left[\mu_{1}\left(\mathbf{Y}_{l}-\hat{\mathbf{Y}}_{l}\right)^{\top} \mathbf{S}\left(\mathbf{Y}_{l}-\hat{\mathbf{Y}}_{l}\right)+\mu_{2} \hat{\mathbf{Y}}_{l}^{T} \mathbf{L} \hat{\mathbf{Y}}_{l}+\mu_{3}\left\|\hat{\mathbf{Y}}_{l}-\mathbf{R}_{l}\right\|_{2}^{2}\right] .
$$

The objective in Equation 5] is similar to the Quadratic Cost Criteria [3], with the exception that the matrices $\mathbf{S}$ and $\mathbf{L}$ have different constructions. We remind the reader that $\hat{\mathbf{Y}}_{l}, \mathbf{Y}_{l}, \mathbf{R}_{l}$ are the 1 th columns (each of size $n \times 1$ ) of the matrices $\hat{\mathbf{Y}}, \mathbf{Y}$ and $\mathbf{R}$ respectively.

\subsection{Solving the Optimization Problem}

We now develop an algorithm to optimize (5] similar to the quadratic cost criteria [3]. Differentiating Equation 5 w.r.t. $\hat{\mathbf{Y}}_{l}$ we get,

$$
\begin{aligned}
\frac{1}{2} \frac{\delta C\left(\hat{\mathbf{Y}}_{)}\right.}{\delta \hat{\mathbf{Y}}_{l}} & =\mu_{1} \mathbf{S}\left(\hat{\mathbf{Y}}_{l}-\mathbf{Y}_{l}\right)+\mu_{2} \mathbf{L} \hat{\mathbf{Y}}_{l}+\mu_{3}\left(\hat{\mathbf{Y}}_{l}-\mathbf{R}_{l}\right) \\
& =\left(\mu_{1} \mathbf{S}+\mu_{2} \mathbf{L}+\mu_{3} \mathbf{I}\right) \hat{\mathbf{Y}}_{l}-\left(\mu_{1} \mathbf{S} \mathbf{Y}_{l}+\mu_{3} \mathbf{R}_{l}\right)
\end{aligned}
$$

Differentiating once more we get,

$$
\frac{1}{2} \frac{\delta C\left(\hat{\mathbf{Y}}_{)}\right.}{\delta \hat{\mathbf{Y}}_{l} \delta \hat{\mathbf{Y}}_{l}}=\mu_{1} \mathbf{S}+\mu_{2} \mathbf{L}+\mu_{3} \mathbf{I}
$$

and since both $\mathbf{S}$ and $\mathbf{L}$ are symmetric and positive semidefinite matrices (PSD), we get that the Hessian is PSD as well. Hence, the optimal minima is obtained by setting the first derivative (i.e. Equation (6) ) to 0 as follows,

$$
\left(\mu_{1} \mathbf{S}+\mu_{2} \mathbf{L}+\mu_{3} \mathbf{I}\right) \hat{\mathbf{Y}}_{l}=\left(\mu_{1} \mathbf{S} \mathbf{Y}_{l}+\mu_{3} \mathbf{R}_{l}\right) .
$$

Hence, the new labels $(\hat{\mathbf{Y}})$ can be obtained by a matrix inversion followed by matrix multiplication. However, this can be quite expensive when large matrices are involved. A more efficient way to obtain the new label scores is to solve a set of linear equations using Jacobi iteration which we now describe.

\subsection{Jacobi Method}

Given the following linear system (in $x$ )

$$
\mathbf{M} x=b
$$

the Jacobi iterative algorithm defines the approximate solution at the $(\mathrm{t}+1)$ th iteration given the solution at $t h$ iteration as follows,

$$
x_{i}^{(t+1)}=\frac{1}{\mathbf{M}_{i i}}\left(b_{i}-\sum_{j \neq i} \mathbf{M}_{i j} x_{j}^{(t)}\right) .
$$


We apply the iterative algorithm to our problem by substituting $x=\hat{\mathbf{Y}}_{l}, \mathbf{M}=\mu_{1} \mathbf{S}+$ $\mu_{2} \mathbf{L}+\mu_{3} \mathbf{I}$ and $b=\mu_{1} \mathbf{S} \mathbf{Y}_{l}+\mu_{3} \mathbf{R}_{l}$ in (7),

$$
\hat{\mathbf{Y}}_{v l}^{(t+1)}=\frac{1}{\mathbf{M}_{v v}}\left(\mu_{1}\left(\mathbf{S Y}_{l}\right)_{v}+\mu_{3} \mathbf{R}_{v l}-\sum_{u \neq v} \mathbf{M}_{v u} \hat{\mathbf{Y}}_{u l}^{(t)}\right)
$$

Let us compute the values of $\left(\mathbf{S Y}_{l}\right)_{i}, \mathbf{M}_{i j(j \neq i)}$ and $\mathbf{M}_{i i}$. First,

$$
\mathbf{M}_{v u(v \neq u)}=\mu_{1} \mathbf{S}_{v u}+\mu_{2} \mathbf{L}_{v u}+\mu_{3} \mathbf{I}_{v u} .
$$

Note that since $\mathbf{S}$ and $\mathbf{I}$ are diagonal, we have that $\mathbf{S}_{v u}=0$ and $\mathbf{I}_{v u}=0$ for $u \neq v$. Substituting the value of $\mathbf{L}$ we get,

$$
\mathbf{M}_{v u(v \neq u)}=\mu_{2} \mathbf{L}_{v u}=\mu_{2}\left(\mathbf{D}_{v u}+\overline{\mathbf{D}}_{v u}-\mathbf{W}_{v u}^{\prime}-\mathbf{W}_{u v}^{\prime}\right),
$$

and as before the matrices $\mathbf{D}$ and $\overline{\mathbf{D}}$ are diagonal and thus $\mathbf{D}_{v u}+\overline{\mathbf{D}}_{v u}=0$. Finally, substituting the values of $\mathbf{W}_{v u}^{\prime}$ and $\mathbf{W}_{u v}^{\prime}$ we get,

$$
\mathbf{M}_{v u(v \neq u)}=-\mu_{2} \times\left(p_{v}^{\text {cont }} \times \mathbf{W}_{v u}+p_{u}^{\text {cont }} \times \mathbf{W}_{u v}\right) .
$$

We now compute the second quantity,

$$
\left(\mathbf{S Y}_{l}\right)_{v u}=\mathbf{S}_{v v} \mathbf{Y}_{v v}+\sum_{t \neq v} \mathbf{S}_{v t} \mathbf{Y}_{t u}=p_{v}^{\mathrm{i} n j} \times \mathbf{Y}_{v v}
$$

where the second term equals zero since $\mathbf{S}$ is diagonal. Finally, the third term,

$$
\begin{aligned}
\mathbf{M}_{v v} & =\mu_{1} \mathbf{S}_{v v}+\mu_{2} \mathbf{L}_{v v}+\mu_{3} \mathbf{I}_{v v} \\
& =\mu_{1} \times p_{v}^{\mathrm{i} n j}+\mu_{2}\left(\mathbf{D}_{v v}+\overline{\mathbf{D}}_{v v}-\mathbf{W}_{v v}^{\prime}-\mathbf{W}_{v v}^{\prime}\right)+\mu_{3} \\
& =\mu_{1} \times p_{v}^{\mathrm{inj}}+\mu_{2} \sum_{u \neq v}\left(p_{v}^{\text {cont }} \mathbf{W}_{v u}+p_{u}^{\text {cont }} \mathbf{W}_{u v}\right)+\mu_{3} .
\end{aligned}
$$

Plugging the above equations into $(8)$ and using the fact that the diagonal elements of $\mathbf{W}$ are zero, we get,

$$
\hat{\mathbf{Y}}_{v}^{(t+1)}=\frac{1}{\mathbf{M}_{v v}}\left(\mu_{1} p_{v}^{\text {inj }} \mathbf{Y}_{v}+\mu_{2} \sum_{u}\left(p_{v}^{\text {cont }} \mathbf{W}_{v u}+p_{u}^{\text {cont }} \mathbf{W}_{u v}\right) \hat{\mathbf{Y}}_{u}^{(t)}+\mu_{3} p_{v}^{\text {abnd }} \mathbf{r}\right) .
$$

We call the new algorithm MAD for Modified-Adsorption and it is summarized in Algorithm 2. Note that for graphs $G$ that are invariant to permutations of the vertices, and setting $\mu_{1}=2 \times \mu_{2}=\mu_{3}=1$, MAD reduces to the Adsorption algorithm.

\subsection{Convergence}

A sufficient condition for the iterative process of Equation (7) to converge is that $\mathbf{M}$ is strictly diagonally dominant [10], that is if,

$$
\left|\mathbf{M}_{v v}\right|>\sum_{u \neq v}\left|\mathbf{M}_{v u}\right| \quad \text { for all values of } v
$$




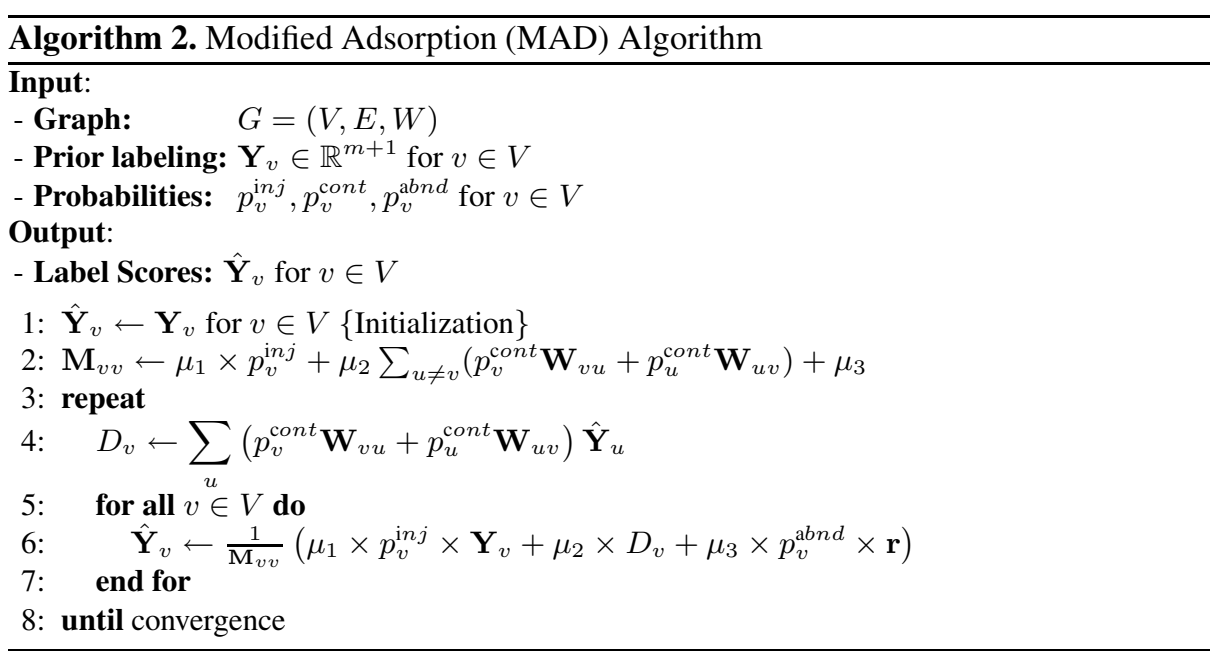

We have,

$$
\begin{aligned}
\left|\mathbf{M}_{v v}\right|-\sum_{u \neq v}\left|\mathbf{M}_{v u}\right| & =\mu_{1} \times p_{v}^{\mathrm{i} n j}+\mu_{2} \times \sum_{u \neq v}\left(p_{v}^{\mathrm{cont}} \times \mathbf{W}_{v u}+p_{u}^{\mathrm{cont}} \times \mathbf{W}_{u v}\right)+\mu_{3}- \\
\mu_{2} \times \sum_{u \neq v}\left(p_{v}^{\mathrm{cont}} \times \mathbf{W}_{v u}+p_{u}^{\mathrm{cont}} \times \mathbf{W}_{u v}\right) & \\
& =\mu_{1} \times p_{v}^{\mathrm{i} n j}+\mu_{3}
\end{aligned}
$$

Note that $p_{v}^{\mathrm{inj}} \geq 0$ for all $v$ and that $\mu_{3}$ is a free parameter in 111. Thus we can guarantee a strict diagonal dominance (and hence convergence) by setting $\mu_{3}>0$.

\section{Extensions: Non-mutually Exclusive Labels}

In many learning settings, labels are not mutually exclusive. For example, in hierarchical classification, labels are organized in a tree. In this section, we extend the MAD algorithm to handle dependence among labels. This can be easily done using our new formulation which is based on objective optimization. Specifically, we shall add additional terms to the objective for each pair of dependent labels. Let $C$ be a $m \times m$ matrix where $m$ is the number of labels (excluding the dummy label) as before. Each entry, $C_{l l^{\prime}}$, of this matrix $C$ represents the dependence or similarity among the labels $l$ and $l^{\prime}$. By encoding dependence in this pairwise fashion, we can capture dependencies among labels represented as arbitrary graphs. The extended objective is shown in Equation 12

$$
\begin{gathered}
C(\hat{\mathbf{Y}})=\sum_{l}\left[\mu_{1}\left(\mathbf{Y}_{l}-\hat{\mathbf{Y}}_{l}\right)^{\top} \mathbf{S}\left(\mathbf{Y}_{l}-\hat{\mathbf{Y}}_{l}\right)+\mu_{2} \hat{\mathbf{Y}}_{l}^{T} \mathbf{L} \hat{\mathbf{Y}}_{l}+\mu_{3}\left\|\hat{\mathbf{Y}}_{l}-\mathbf{R}_{l}\right\|_{2}^{2}\right. \\
\left.+\mu_{4} \sum_{i} \sum_{l, l^{\prime}} C_{l l^{\prime}}\left(\hat{\mathbf{Y}}_{i l}-\hat{\mathbf{Y}}_{i l^{\prime}}\right)^{2}\right]
\end{gathered}
$$


The last term in Equation 12 penalizes the algorithm if similar labels (as determined by the matrix $C$ ) are assigned different scores, with severity of the penalty controlled by $\mu_{4}$. Now, analyzing the objective in Equation 12 in the manner outlined in Section 4 we arrive at the update rule shown in Equation 13.

$$
\begin{array}{r}
\hat{\mathbf{Y}}_{v l}^{(t+1)}=\frac{1}{\mathbf{M}_{v v}^{l}}\left(\mu_{1} p_{v}^{\mathrm{i} n j} \mathbf{Y}_{v l}+\mu_{2} \sum_{u}\left(p_{v}^{\mathrm{cont}} \mathbf{W}_{v u}+p_{u}^{\mathrm{cont}} \mathbf{W}_{u v}\right) \hat{\mathbf{Y}}_{u l}^{(t)}+\right. \\
\left.\mu_{3} p_{v}^{\mathrm{a} b n d} \mathbf{r}_{l}+\mu_{4} \sum_{l^{\prime}} C_{l l^{\prime}} \hat{\mathbf{Y}}_{i l^{\prime}}\right)
\end{array}
$$

where,

$$
\mathbf{M}_{v v}^{l}=\mu_{1} \times p_{v}^{\mathrm{i} n j}+\mu_{2} \times \sum_{u \neq v}\left(p_{v}^{\mathrm{cont}} \mathbf{W}_{v u}+p_{u}^{\mathrm{cont}} \mathbf{W}_{u v}\right)+\mu_{3}+\mu_{4} \sum_{l^{\prime}} C_{l l^{\prime}}
$$

Replacing Line 6 in MAD (Algorithm 2) with Equation 13, we end up with a new algorithm: Modified Adsorption for Dependent Labels (MADDL). In Section 6.4, we shall use MADDL to obtain smooth ranking for sentiment classification.

\section{Experimental Results}

We compare MAD with various state-of-the-art learning algorithms on two tasks, text classification (Sec. 6.1) and sentiment analysis (Sec. 6.2), and demonstrate its effectiveness. In Sec. 6.3, we also provide experimental evidence showing that MAD is quite insensitive to wide variation of values of its hyper-parameters. In Sec. 6.4 we present evidence showing how MADDL can be used to obtain smooth ranking for sentiment prediction, a particular instantiation of classification with non-mutually exclusive labels. For the experiments reported in this section involving Adsorption, MAD and MADDL, the a-priori label matrix $\mathbf{Y}$ was column-normalized so that all labels have equal overall injection score. Also, the dummy label was ignored during evaluation as its main role is to add regularization during learning phase only.

\subsection{Text Classification}

World Wide Knowledge Base (WebKB) is a text classification dataset widely used for evaluating transductive learning algorithms. Most recently, the dataset was used by Subramanya and Bilmes [11], who kindly shared their preprocessed complete WebKB graph with us. There are a total of 4,204 vertices in the graph, with the nodes labeled with one of four categories: course, faculty, project, student. A K-NN graph is created from this complete graph by retaining only top $K$ neighbors of each node, where the value of $K$ is treated as a hyper-parameter.

We follow the experimental protocol in [11]. The dataset was randomly partitioned into four sets. A transduction set was generated by first selecting one of the four splits at random and then sampling $n_{l}$ documents from it; the remaining three sets are used as the test set for evaluation. This process was repeated 21 times to generate as many trainingtest splits. The first split was used to tune the hyper-parameters, with search over the 
Table 1. PRBEP for the WebKB data set with $n_{l}=48$ training and 3148 testing instances. All results are averages over 20 randomly generated transduction sets. The last row is the macroaverage over all the classes. MAD is the proposed approach. Results for SVM, TSVM, SGT, LP and AM are reproduced from Table 2 of [11].

\begin{tabular}{|l|c|c|c|c|c|c|c|}
\hline Class & SVM & TSVM & SGT & LP & AM & Adsorption & MAD \\
\hline \hline course & 46.5 & 43.9 & 29.9 & 45.0 & $\mathbf{6 7 . 6}$ & 61.1 & 67.5 \\
faculty & 14.5 & 31.2 & 42.9 & 40.3 & 42.5 & $\mathbf{5 2 . 8}$ & 42.2 \\
project & 15.8 & 17.2 & 17.5 & 27.8 & 42.3 & $\mathbf{5 2 . 6}$ & 45.5 \\
student & 15.0 & 24.5 & 56.6 & 51.8 & 55.0 & 39.8 & $\mathbf{5 9 . 6}$ \\
\hline \hline average & 23.0 & 29.2 & 36.8 & 41.2 & 51.9 & 51.6 & $\mathbf{5 3 . 7}$ \\
\hline
\end{tabular}

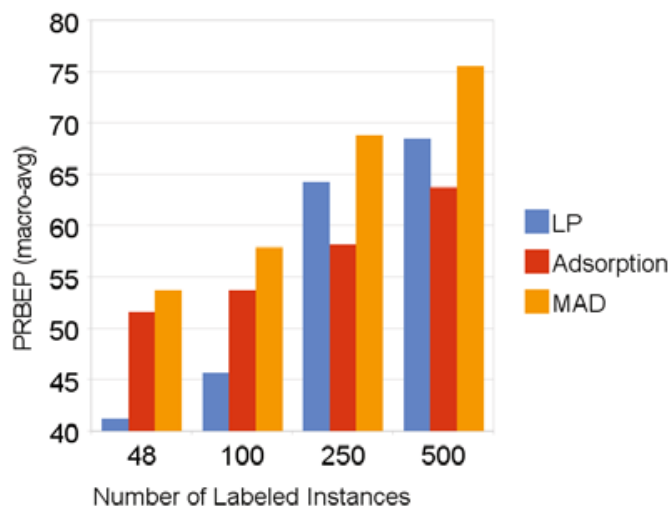

Fig. 1. PRBEP (macro-averaged) for the WebKB dataset with 3148 testing instances. All results are averages over 20 randomly generated transduction sets.

following: $K \in\{10,50,100,500,1000,2000,4204\}, \mu_{2}, \mu_{3} \in\{1 e-8,1 e-4,1 e-2,1$, $10,1 e 2,1 e 3\}$. The value of $\mu_{1}$ was set to 1 for this experiment. Both for Adsorption and MAD, the optimal value of $K$ was 1,000. Furthermore, the optimal value for the other parameters were found to be $\mu_{2}=\mu_{3}=1$. As in previous work [11], we use Precision-Recall Break Even Point (PRBEP) [9] as the evaluation metric. Same evaluation measure, dataset and the same experimental protocol makes the results reported here directly comparable to those reported previously [11]. For easier readability, the results from Table 2 of Subramanya and Bilmes [11] are cited in Table 1] of this paper, comparing performance of Adsorption based methods (Adsorption and MAD) to many previously proposed approaches: SVM [6], Transductive-SVM [6], Spectral Graph Transduction (SGT) [7], Label Propagation (LP) [16] and Alternating Minimization (AM) [11]. The first four rows in Table 1 shows PRBEP for individual categories, with the last line showing the macro-averaged PRBEP across all categories. The MAD algorithm achieves the best performance overall (for $n_{l}=48$ ).

Performance comparison of MAD and Adsorption for increasing $n_{l}$ are shown in Figure 11 Comparing these results against Fig. 2 in Subramanya and Bilmes [11], it seems that MAD outperforms all other methods compared (except AM [11]) for all 


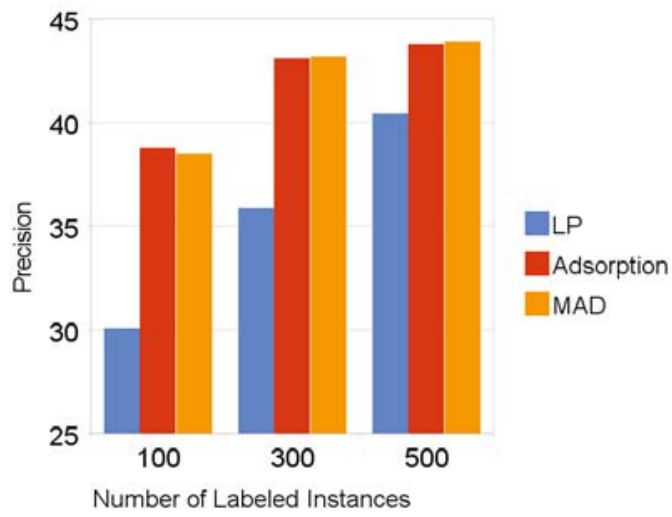

Fig. 2. Precision for the Sentiment Analysis dataset with 3568 testing instances. All results are averages over 4 randomly generated transduction sets.

Table 2. Average prediction loss at ranks $1 \& 2$ (for various values of $\mu_{4}$ ) for sentiment prediction. All results are averaged over 4 runs. See Section 6.4 for details.

\begin{tabular}{|l|c|c|c|c|c|c|}
\hline & \multicolumn{6}{|c|}{$\mu_{4}$} \\
\cline { 2 - 7 } & 0 & 1 & 10 & 100 & $1 \mathrm{e} 3$ & $1 \mathrm{e} 4$ \\
\hline \hline Prediction Loss (L1) at rank 1 & 0.93 & 0.93 & 0.92 & 0.90 & 0.90 & 0.90 \\
Prediction Loss (L1) at rank 2 & 1.21 & 1.20 & 1.12 & 0.96 & 0.97 & 0.97 \\
\hline
\end{tabular}

values of $n_{l}$. MAD performs better than AM for $n_{l}=48$, but achieves second best solution for the other three values of $n_{l}$. We are currently investigating why MAD is best for settings with fewer labeled examples.

\subsection{Sentiment Analysis}

The goal of sentiment analysis is to automatically assign polarity scores to text collections, with a high score reflecting positive sentiment (user likes) and a low score reflecting negative sentiment (user dislikes). In this section, we report results on sentiment classification in the transductive setting. From Section 6.1 and [11], we observe that Label Propagation (LP) [16] is one of the best performing L2-norm based transductive learning algorithm. Hence, we compare the performance of MAD against Adsorption and LP.

For the experiments in this section, we use a set of 4,768 user reviews from the electronics domain [4]. Each review is assigned one of the four scores: 1 (worst), 2, 3, 4 (best). We create a K-NN graph from these reviews by using cosine similarity as the measure of similarity between reviews. We created 5 training-test splits from this data using the process described in Section 6.1. One split was used to tune the hyper-parameters while the rest were used for training and evaluation. Hyper-parameter search was carried over the following ranges: $K \in\{10,100,500\}, \mu_{1} \in\{1,100\}$, 


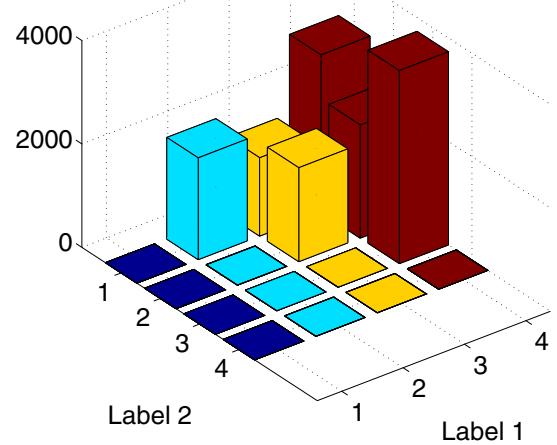

Fig. 3. Plot of counts of top predicted label pairs (order ignored) in MAD's predictions with $\mu_{1}=\mu_{2}=1, \mu_{3}=$ 100

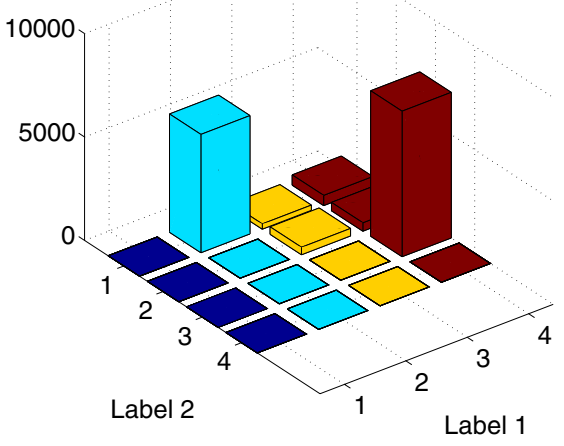

Fig. 4. Plot of counts of top label pairs (order ignored) in MADDL's predictions (Section 5), with $\mu_{1}=\mu_{2}=1$, $\mu_{3}=100, \mu_{4}=1 e 3$

$\mu_{2} \in\{1 e-4,1,10\}, \mu_{3} \in\{1 e-8,1,100,1 e 3\}$. Precision is used as the evaluation metric. Comparison of different algorithms for varying number of labeled instances are shown in Figure 2. From this, we note that MAD and Adsorption outperform LP, while Adsorption and MAD are competitive.

\subsection{Parameter Sensitivity}

We evaluated the sensitivity of MAD to variations of its $\mu_{2}$ and $\mu_{3}$ hyper-parameters, with all other hyper-parameters fixed. We used a 2000-NN graph constructed from the WebKB dataset and a 500-NN graph constructed from the Sentiment dataset. In both cases, 100 nodes were labeled. We tried three values each for $\mu_{2}$ and $\mu_{3}$, ranging in at least 3 order of magnitude. For the WebKB, the PRBEP varied between $43.1-49.9$ and for the sentiment data, the precision varied in the range $31.4-36.4$ with $\mu_{2} \leq \mu_{3}$ while precision dropped to 25 with $\mu_{2}>\mu_{3}$. This underscores the need for regularization in these models, which is enforced with high $\mu_{3}$. We note that in both cases the algorithm is less sensitive to the value of $\mu_{2}$ than the value of $\mu_{3}$. In general, we have found that setting $\mu_{3}$ to one or two order magnitude more than $\mu_{2}$ is a reasonable choice. We have also found that the MAD algorithm is quite insensitive to variations in $\mu_{1}$. For example on the sentiment dataset, we tried two values for $\mu_{1}$ ranging two order of magnitude, with other hyper-parameters fixed. In this case, precision varied in the range 36.2 - 36.3.

\subsection{Smooth Ranking for Sentiment Analysis}

We revisit the sentiment prediction problem in Section 6.2, but with the additional requirement that ranking of the labels $(1,2,3,4)$ generated by the algorithm should be 
smooth i.e. we prefer the ranking $1>2>3>4$ over the ranking $1>4>3>2$, where $3>2$ means that the algorithm ranks label 3 higher than label 2 . The ranking $1>2>$ $3>4$ is smoother as it doesn't involve rough transition $1>4$ which is present in $1>4$ $>3>2$. We use the framework of stating requirements as an objective to be optimized. We use the MADDL algorithm of Sec.5 5 initializing the matrix $C$ as follows (assuming that labels 1 and 2 are related, while labels 3 and 4 are related):

$$
C_{12}=C_{21}=1 \quad, \quad C_{34}=C_{43}=1
$$

with all other entries in matrix $C$ set to 0 . Such constraints (along with appropriate $\mu_{4}$ in Equation (12) will force the algorithm to assign similar scores to dependent labels, thereby assigning them adjacent ranks in the final output. MAD and MADDL were then used to predict ranked labels for vertices on a 1000-NN graph constructed from the sentiment data used in Sec.6.2, with 100 randomly selected nodes labeled. For this experiment we set $\mu_{1}=\mu_{2}=1, \mu_{3}=100$. The $L 1$-loss between the gold label and labels predicted at ranks $r=1,2$ for increasing values of $\mu_{4}$ are given in Table 2 Note that, MADDL with $\mu_{4}=0$ corresponds to MAD. From Table 2 we observe that with increasing $\mu_{4}$, MADDL is ranking at $r=2$ a label which is related (as per $C$ ) to the top ranked label at $r=1$, but at the same time maintain the quality of prediction at $r=1$ (first row of Table 2), thereby ensuring a smoother ranking. From Table 2, we also observe that MADDL is insensitive to variations of $\mu_{4}$ beyond a certain range. This suggests that $\mu_{4}$ may be set to a (high) value and that tuning it may not be necessary.

Another view of the same phenomenon is shown in Fig. 3 and Fig. 4 In these figures, we plot the counts of top predicted label pair (order of prediction is ignored for better readability) generated by the MAD and MADDL algorithms. By comparing these two figures we observe that label pairs (e.g. $(2,1)$ and $(4,3))$ favored by $C$ (above) are more frequent in MADDL's predictions than in MAD's. At the same time, non-smooth predictions (e.g. $(4,1))$ are virtually absent in MADDL's predictions while they are quite frequent in MAD's. These clearly demonstrate MADDL's ability to generate smooth predictions in a principled way, and more generally the ability to handle data with nonmutually exclusive or dependent labels.

\section{Related Work}

LP [16] is one of the first graph based semi-supervised algorithms. Even though there are several similarities between LP and MAD, there are important differences: (1) LP doesn't allow the labels on seeded nodes to change (while MAD does). As was pointed out previously [3], this can be problematic in case of noisy seeds. (2) There is no way for LP to express label uncertainty about a node. MAD can accomplish this by assigning high score to the dummy label. More recently, a KL minimization based algorithm was presented in [11]. Further investigation is necessary to determine the merits of each approach. For a general introduction to the area of graph-based semi-supervised learning, the reader is referred to a survey by Zhu [15].

\section{Conclusion}

In this paper we have analyzed the Adsorption algorithm [1] and proposed a new graph based semi-supervised learning algorithm, MAD. We have developed efficient 
(iterative) solution to solve our convex optimization based learning problem. We have also listed the conditions under which the algorithm is guaranteed to converge. Transition to an optimization based learning algorithm allows us to easily extend the algorithm to handle data with non-mutually exclusive labels, resulting in the MADDL algorithm. We have provided experimental evidence demonstrating effectiveness of our proposed methods. As part of future work, we plan to evaluate the proposed methods further and apply the MADDL method in problems with dependent labels (e.g. Information Extraction).

\section{Acknowledgment}

This research is partially supported by NSF grant \#IIS-0513778. The authors would like to thank F. Pereira and D. Sivakumar for useful discussions.

\section{References}

1. Baluja, S., Seth, R., Sivakumar, D., Jing, Y., Yagnik, J., Kumar, S., Ravichandran, D., Aly, M.: Video suggestion and discovery for youtube: taking random walks through the view graph. In: WWW (2008)

2. Belkin, M., Niyogi, P., Sindhwani, V.: Manifold regularization: A geometric framework for learning from labeled and unlabeled examples. JMLR 7, 2399-2434 (2006)

3. Bengio, Y., Delalleau, O., Roux, N.: Label Propogation and Quadratic Criterion. In: SemiSupervised Learning (2007)

4. Blitzer, J., Dredze, M., Pereira, F.: Biographies, bollywood, boom-boxes and blenders: Domain adaptation for sentiment classification. In: ACL (2007)

5. Indyk, P., Matousek, J.: Low-distortion embeddings of finite metric spaces. In: Handbook of Discrete and Computational Geometry (2004)

6. Joachims, T.: Transductive inference for text classification using support vector machines. In: ICML (1999)

7. Joachims, T.: Transductive learning via spectral graph partitioning. In: ICML (2003)

8. Katz, V.J.: The history of stokes' theorem. Mathematics Magazine 52(3), 146-156 (1979)

9. Raghavan, V., Bollmann, P., Jung, G.: A critical investigation of recall and precision as measures of retrieval system performance. ACM TOIS 7(3), 205-229 (1989)

10. Saad, Y.: Iterative Methods for Sparse Linear Systems. Society for Industrial Math. (2003)

11. Subramanya, A., Bilmes, J.: Soft-Supervised Learning for Text Classification. In: EMNLP (2008)

12. Szummer, M., Jaakkola, T.: Partially labeled classification with markov random walks. In: NIPS (2002)

13. Talukdar, P.P., Reisinger, J., Pasca, M., Ravichandran, D., Bhagat, R., Pereira, F.: Weakly supervised acquisition of labeled class instances using graph random walks. In: EMNLP (2008)

14. Wang, J., Jebara, T., Chang, S.: Graph transduction via alternating minimization. In: ICML (2008)

15. Zhu, X.: Semi-supervised learning literature survey (2005)

16. Zhu, X., Ghahramani, Z.: Learning from labeled and unlabeled data with label propagation. Technical report, CMU CALD tech report (2002)

17. Zhu, X., Ghahramani, Z., Lafferty, J.: Semi-supervised learning using gaussian fields and harmonic functions. In: ICML (2003) 